FORSCHUNG - AUSBILDUNG - WEITERBILDUNG

Bericht Nr. 131

A MODEL FOR THE CLOUDINESS OF FABRICS

Joachim/Weickert

UNIVERSITÄT KAISERSLAUTERN

Fachbereich Mathematik

Postfach 3049

D-67653 Kaiserslautern

Februar 1995 


\title{
A Model for the Cloudiness of Fabrics
}

\author{
Joachim Weickert \\ Laboratory of Technomathematics, University of Kaiserslautern, \\ P.O. Box 3049, D-67653 Kaiserslautern, Germany. \\ l'el.: ++49631205 4084, Fax: ++496312053052. \\ weickert@mathematik.uni-kl.de
}

February 10, 1995

\begin{abstract}
Cloudy inhomogenities in artificial fabrics are graded by a fast method which is based on a Laplacian pyramid decomposition of the fabric image. This band-pass representation takes into account the scale character of the cloudiness. A quality measure of the entire cloudiness is obtained as a weighted mean over the variances of all scales.
\end{abstract}




\title{
A Model for the Cloudiness of Fabrics
}

\author{
Joachim Weickert* \\ Laboratory of Technomathematics, University of Kaiserslautern, \\ P.O. Box 3049, D-67653 Kaiserslautern, Germany.
}

\section{Introduction}

A large variety of industrial products ranging from carpets to baby napkins makes use of artificial fabrics. For all these products, the fabric quality plays an important role. It is deteriorated by inhomogenities such as clouds and stripes.

Clouds result from isotropic fibre agglomerations, thus having no preferred directions. Since large clouds do not look very pleasing, the scale of the cloudiness gives a measure for the optical appearance of the fabric. Moreover, the areas with the lowest density characterize the weakest parts of the fabric.

Stripes consist of adjacent fibres preferring a certain direction. Therefore, the orientation of the stripes characterizes the fabric anisotropy, which has a strong impact on its mechanical properties.

The fabric producing company which brought this problem to our attention takes online images of their fabrics with a resolution well-suited for estimating the cloudiness. For evaluating the stripes, more advanced methods have to be applied which require a higher resolution. This cannot be investigated during the production process anymore. In the present paper, we shall therefore focus on the cloudiness.

In order to obtain objective, reliable quality measures for cloudiness, a suitable mathematical model is needed. On one hand, such a model would be useful for the internal assessment during the production, e.g. for surveying the product quality, for determining machine cleaning cycles and for comparing different machines. One the other hand, objective quality criteria provide standards for the customers and help them to specify their demands in a proper way.

\footnotetext{
*supported by a grant from "Stiftung Innnovation des Landes Rheinland-Pfalz"
} 
A good model should be exact enough to match the human perception of cloudiness and it should allow fast algorithms which enable the company to perform grading during the production. In the present paper, we shall discuss such a model.

We can rely our studies on a number of previous approaches in this field.

First it should be observed that simple ideas like calculating the variance or the entropy of an image are not sensitive enough for our purpose, since they are independent of the ordering of the grey values. Hence, they cannot distinguish between multiple small clouds and one large cloud. In both cases, they yield the same amount of inhomogenity. Since for the human observer, there is a big difference between these two cases, it becomes clear, that a suitable model must take into account the scale of a cloud.

Early suggestions in this direction were made by Neunzert and Wetton [4], who proposed to use the discrepancy as a cloudiness criterion. Roughly speaking, the discrepancy measures the largest cloud or the largest hole in the fabric. A fast algorithm for two-dimensional interval discrepancy was presented by Hackh [3]. He reported that the results were still too coarse and not always selective enough for the desired purpose.

Stark [5] took into account the scale character of the cloudiness by analysing the fabric in a wavelet basis. However, his method did not make use of the main feature of wavelets, the localization in frequency and space, thus, it seems that simpler techniques such as Fourier analysis suffice as well. Moreover, the grading step of this model was based on a fractal dimension assumption: it was claimed that the variance depends in a linear way on the scale. Recent experiences do not confirm this hypothesis.

Weickert [6] suggested to process the fabric image using nonlinear anisotropic diffusion in order to visualize clouds and the main stripes simultaneously. Although the results were fairly promising, the proposed method is not yet fast enough for online assessment. Furthermore, there seems to be no need to process both quality relevant features simultaneously.

The purpose of the present paper is to show that linear diffusion suffices for evaluating the cloudiness. The linear diffusion process is used implicitly in the image description by means of a Laplacian pyramid. A Laplacian pyramid can be thought of as a multiscale representation providing a band. pass decomposition of the image. Being a multigrid technique, this method is extremly fast. As a quality functional of the cloudiness, it is proposed to 
use a weighted mean over the variances of all scales, with weights adapted to the human impression of cloudiness.

The paper is organized as follows.

In section 2 , we shall briefly review the concept of image pyramids. We will see that the commonly used Laplacian pyramid has to be modified at the boundaries in order to be suitable for our task. Moreover, we shall point out the differences to other multiscale representations such as Fourier or wavelet analysis. The third section decribes the proposed quality functional for the cloudiness of a fabric and discusses some experiments. In section 4 , the paper concludes with a summary of the obtained results.

\section{Multiscale analysis with the Laplacian pyramid}

\subsection{Gaussian and Laplacian pyramid}

For simplicity, we first restrict ourselves to the one dimensional case in order to sketch the pyramid concept in image processing. Due to its separability, the two dimensional case follows immediately from the one dimensional case (for more details, see Burt and Adelson [2]).

We start by defining a (grey value) image as a vector $u=\left(u_{0}, \ldots, u_{2^{N}}\right)^{T} \in$ $\mathbb{R}^{2^{N}+1}$. We consider the linear interpolation operators

$$
\begin{aligned}
I_{2^{k}+1}^{2^{k+1}+1} & =\left(\begin{array}{ccccccc}
1 & 0 & 0 & \cdots & 0 & 0 & 0 \\
\frac{1}{2} & \frac{1}{2} & 0 & \cdots & 0 & 0 & 0 \\
0 & 1 & 0 & \cdots & 0 & 0 & 0 \\
0 & \frac{1}{2} & \frac{1}{2} & \cdots & 0 & 0 & 0 \\
\cdots & \cdots & \cdots & \cdots & \cdots & \cdots \\
0 & 0 & 0 & \cdots & \frac{1}{2} & \frac{1}{2} & 0 \\
0 & 0 & 0 & \cdots & 0 & 1 & 0 \\
0 & 0 & 0 & \cdots & 0 & \frac{1}{2} & \frac{1}{2} \\
0 & 0 & 0 & \cdots & 0 & 0 & 1
\end{array}\right) \in \mathbb{R}^{\left(2^{k+1}+1\right) \times\left(2^{k}+1\right)} \quad(k=1, \ldots, N-1) \\
I_{1}^{3}= & \left(\begin{array}{l}
1 \\
1 \\
1
\end{array}\right) \in \mathbb{R}^{3 \times 1} .
\end{aligned}
$$


Note that their row sums are always 1.

Furthermore, we define the following restriction operators.

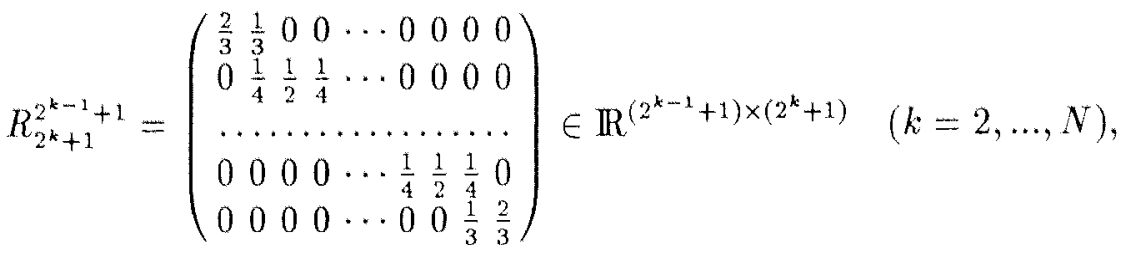

$$
\begin{aligned}
& R_{3}^{1}=\left(\frac{1}{3} \frac{1}{3} \frac{1}{3}\right) \in \mathbb{R}^{1 \times 3} .
\end{aligned}
$$

As for the interpolation operators, their rows sum up to 1 as well. Apart from $R_{3}^{1}$ and boundary points, the restriction operators consist of a convolution with the binomial mask $\left[\frac{1}{1}, \frac{1}{2}, \frac{1}{4}\right]$ followed by a coarser subsampling. In multigrid analysis, they are also called full weighting operators [1, page 39]. The preceding convolution mask is nothing else but an explicit finite difference scheme to the diffusion equation $u_{t}=u_{x x}$ with mesh ratio $\frac{\Delta t}{(\Delta x)^{2}}=\frac{1}{4}$. This scheme is well-known to inherit several important properties of the contimuous diffusion equation such as nonnegativity, maximum-minimum principle, monotonicity preserving, and total variation diminishing (TVD).

Using the preceding family of restriction operators, we define the Gaussian pyramid $\left\{v^{2^{N}+1}, \ldots, v^{1}\right\}$ of $u$ by

$$
\begin{aligned}
v^{2^{N}+1} & =u, \\
v^{2^{N-1}+1} & =R_{2^{N+1}+1}^{2^{N+1}} v^{2^{N}+1}, \\
v^{2^{N-2}+1} & =R_{2^{N-1}+1}^{2^{N-2}+1} v^{2^{N-1}+1}, \\
& \vdots \\
v^{1} & =R_{3}^{1} v^{3} .
\end{aligned}
$$

Due to the coarser subsampling and the smoothing binomial mask, the Gaussian pyramid can be regarded as a sequence of low-pass filtered versions of $u$, whose size is reduced in each step by a factor which is close to $1 / 2$. (Note that the upper index is not an exponent, it denotes the size of the vector.) So if we interpolate every successor in the Gaussian pyramid and subtract it from its predecessor, we get a band-pass representation of $u$ :

$$
\begin{aligned}
w^{2^{N}+1} & =v^{2^{N}+1}-I_{2^{N-1}+1}^{2^{N}+1} v^{2^{N-1}+1} \\
w^{2^{N-1}+1} & =v^{2^{N-1}+1}-I_{2^{N-2}+1}^{2^{N-1}+1} v^{2^{N-2}+1}
\end{aligned}
$$




$$
\begin{aligned}
& w^{3}=v^{3}-I_{1}^{3} v^{1}, \\
& w^{1}=v^{1} .
\end{aligned}
$$

$\left\{w^{2^{N}+1}, \ldots, w^{1}\right\}$ is called Laplacian pyramid of $u$. Its highest frequency component is $w^{2^{N}+1}$.

\section{$2.2 \quad$ Modification at the boundaries}

The paper of Burt and Adelson [2] does not address the question of how to choose the restriction operator at the boundaries. Other choices than the one that we have already seen may cause undesirable effects near the boundaries of the Laplacian pyramid (such as strong oscillations), and the pyramid levels $w^{2^{N}+1}, \ldots, w^{3}$ may not have zero mean. The latter phenomenon is rather untypical for other band-pass representations such as Fourier or wavelet based ones. It can only happen if the restriction with the subsequent interpolation does alter the average grey value of the image. This is also undesirable if we think of the diffusion interpretation of this process: diffusion relies upon the continuity equation and, therefore, it is conservative. This indicates that the restriction operator has to be chosen very carefully at the boundaries in order to fit the interpolation operator well.

The following lemma shows how both operators should be related to ensure that the Laplacian pyramid levels $w^{2^{N}+1}, \ldots, w^{3}$ have zero mean.

Lemma 1 Let all row sums of $A:=\left(a_{i j}\right) \in \mathbb{R}^{m \times n}$ and $B:=\left(b_{i j}\right) \in \mathbb{R}^{n \times m}$ be 1 . Furthermore, assume that $\sum_{k=1}^{m} a_{k j} \neq 0$ and $b_{j i}=a_{i j} / \sum_{k=1}^{m} a_{k j}$ for all $i=1, \ldots, m$, and $j=1, \ldots, n$.

Then for all $x, y \in \mathbb{R}^{m}$ with $y=A B x$, we have $\sum_{i=1}^{m} y_{i}=\sum_{i=1}^{m} x_{i}$.

Proof: Let $C:=\left(c_{i j}\right):=A B$. Since

$$
c_{i j}=\sum_{p=1}^{n} a_{i p} b_{p j}=\sum_{p=1}^{n} \frac{a_{i p} a_{j p}}{\sum_{k=1}^{m} a_{k p}}=\sum_{p=1}^{n} a_{j p} b_{p i}=c_{j i}
$$

for all $i, j \in\{1, \ldots, m\}$, we know that $\mathrm{C}$ is symmetric. Furthermore, the product of two matrices with row sums 1 yields also a matrix whose rows 
sum up to 1 . Thus, all colum sums of $C$ are also 1 , and we end up with the assertion

$$
\sum_{i=1}^{m} y_{i}=\sum_{i=1}^{m} \sum_{j=1}^{m} c_{i j} x_{j}=\sum_{j=1}^{m}\left(\sum_{i=1}^{m} c_{i j}\right) x_{j}=\sum_{j=1}^{m} x_{j} .
$$

We observe that the suggested interpolation and restriction operators satisfy the requirements of the preceding lemma.

It should be noted that - in our case - a correct treatment at the boundaries is of crucial importance. As we will see in section 3 , we need the variance of each pyramid level in order to grade the cloudiness. Especially at coarse levels (corresponding to large clouds), boundary pixels cause an important contribution to this value. Incorrect boundary treatment would be the source of significant deviations and misinterpretations.

\subsection{Comparison with other multiscale methods}

Like Fourier and wavelet transformation, the Laplacian pyramid gives a complete image representation which allows to retrieve the image entirely, see e.g. [2]. Since this representation is completely performed in the spatial domain, we obtain the band-pass filtered versions immcdiatcly, no preceding reconstruction is necessary. In contrast to Fast Fourier Transform (FFT), the computational (and storage) effort is linear in the number of pixels. Thus, on a fast PC or a small workstation, the computation of the Laplacian pyramid is fast enough for online analysis of cloudiness in fabrics. On the other hand, a Laplacian pyramid contains always some redundancy, since the pyramid representation is about $1 / 3$ larger than the original image. The frequency separation between neighbouring scales is not as strict as in the wavelet and Fourier case. Nevertheless, we shall see that it is still sufficient for our purposes. Our experiments have indicated that the Laplacian pyramid is sufficiently robust with respect to translations (a frequent problem when using wavelets) and it performs better at boundaries than wavelet and Fourier methods. A correct boundary treatment would be one of the main problems when using wavelet analysis. For the FFT, discontinuities at the periodic extensions of images may lead to artefacts deceiving anisotropy, which is not apparent in the original image.

Recapping, besides its simplicity and velocity, the robust behaviour near boundarics is the main reason for preferring Laplacian pyramids to other multiscale approaches for the present problem. 


\section{The quality functional for cloudiness}

Having a band-pass representation of the fabric image by means of the Laplacian pyranid, one may take the variance $\sigma_{k}^{2}$ at some scale $k$ as a measure for the cloudiness at this scale. Since our Laplacian pyramid levels have zero mean, the variance is given by

$$
\sigma_{k}^{2}(u)=\frac{1}{2^{k}+1} \sum_{i=0}^{2^{k}}\left(w_{i}^{2^{k}+1}(u)\right)^{2} \quad(k=1, \ldots, N) .
$$

Now the question arises of how to assemble the variances $\sigma_{k}^{2}, k=1, \ldots, N$ to a single value describing the human impression of cloudiness. One of the simplest ideas is to calculate a weighted mean. But how shall the weights be chosen?

To solve this question, an experiment was performed: 18 members of the Laboratory of Technomathematics (with different ages, nationalities, and degrees of expertise) were asked to classify nine fabric images according to their visual appearance of cloudiness. The result was fairly surprising: most of the candidates gave a very similar ranking. This indicates that there seems to exist a typical human impression of cloudiness independent of age, sex, cultural background and knowledge. It takes into account mainly inhomogenities at middle scales. Smaller and larger clouds had significantly less influence to the assessment. For this reason, we may choose weights proportional to a Gaussian distribution which is centered around the middle scales and decreases rapidly towards small and large scales:

$$
w(k)=\frac{\exp \left(-\frac{(k-\mu)^{2}}{2 \sigma^{2}}\right)}{\sum_{j=1}^{N} \exp \left(-\frac{(j-\mu)^{2}}{2 \sigma^{2}}\right)} .
$$

The mean $\mu$ and the standard deviation $\sigma$ are determined in order to fit the experimental data best. Thus, the final expression of the cloudiness $c$ of a fabric $u$ is

$$
c(u)=\sum_{k=1}^{N} w(k) \sigma_{k}^{2}(u)
$$

The validity of the quality functional was checked by comparing its results with the preceding test. Moreover, a simultaneous test was made with 27 
employees of the fabric producing company. In both cases, the ranking of the model was within the standard deviation of ranking of the hunan evaluators. Also with other image sets, similar results could be obtained.

\section{$4 \quad$ Summary and outlook}

The cloudiness of fabrics is a scale-phenomenon which can be analysed sufficiently well using a Laplacian pyramid decomposition. The pyramid should be modified at boundaries in order to reduce errors induced by these values. As a measure of cloudiness, one may use a weighted average of the variances at all scales, with weights according to the human perception. The obtained model reaches the qualities of a human assessor, and it is fast enough for online grading. Therefore, future research can focus on the second quality relevant feature of fabrics, the grading of stripes.

\section{References}

[1] Briggs, W.L.: A multigrid tutorial. SIAM, Philadelphia, 1987

[2] Burt, P.J.; Adelson, E.H.: The Laplacian pyramid as a compact image code. IEEE Trans. Comm. 31 (1983) $532-540$

[3] Hackh, P.: Quality control of artificial fabrics. Report no. 45, Laboratory of 'Technomathematics, University of Kaiserslautern, P.O. Box 3049, 67653 Kaiserslautern, Germany, 1990

[4] Neunzert, H.; Wetton, B.: Pattern recognition using measure space metrics. Report no. 28, Laboratory of Terhnomathematics, University of Kaiserslautern, P.O. Box 3049, 67653 Kaiserslautern, Germany, 1987

[5] Stark, H.-G.: Multiscale analysis, wavelets and texture quality. Report no. 41, Laboratory of Technomathematics, University of Kaiserslautern, P.O. Box 3049, 67653 Kaiserslautern, Germany, 1990

[6] Weickert, J.: Anisotropic diffusion filters for image processing based quality control. In Fasano, A.; Primicerio, M. (Eds.): Proc. of the Seventh European Conf. on Mathematics in Industry. Teubner, Stuttgart, 355-362, 1994 